\title{
Mitral annular calcification
}

\author{
Ragesh Panikkath MD, Deepa Panikkath MD
}

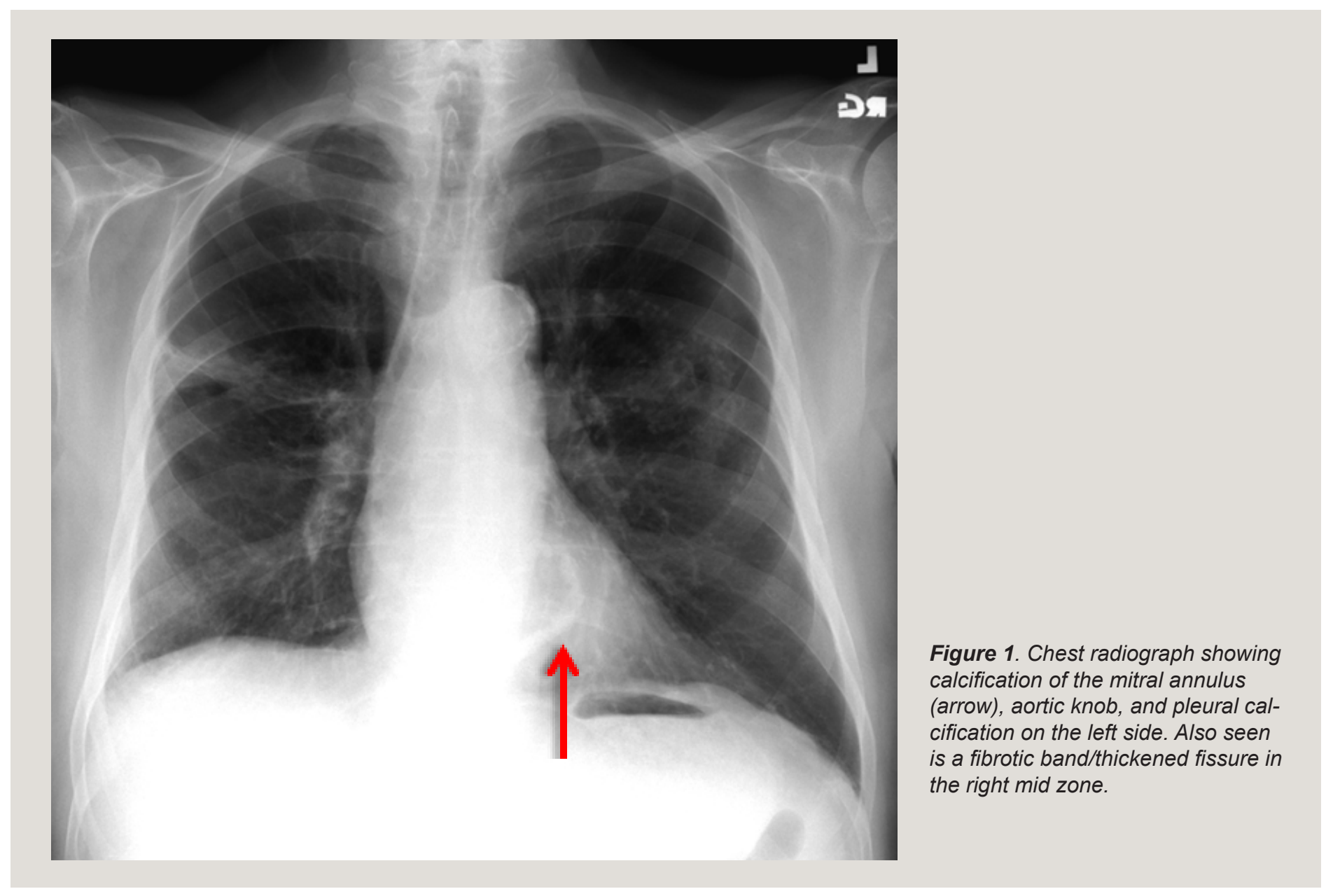

A 79-year-old man with systemic hypertension and stage 3 chronic kidney disease presented with a productive cough for 10 days. He had a history of coronary artery disease, renal artery stenosis, and surgical repair of an abdominal aortic aneurysm and had had a right lower lobe pneumonia 6 months pre-

Corresponding author: Ragesh Panikkath MD

Contact Information: ragesh.panikkath@ttuhsc.edu DOI: 10.12746/swrccc 2014.0206.070 viously. His examination was significant for rales on the right side. A chest radiograph ruled-out recurrent pneumonia. This study showed mitral annular calcification, calcification of the aortic knob, pleural calcification, and a fibrotic band on the right side. He was treated with antibiotics for a lower respiratory tract infection.

The mitral annulus is the " $c$ " shaped fibrous tissue at the left atrio-ventricular junction to which the mitral leaflets are attached. It is absent anteriorly where the 


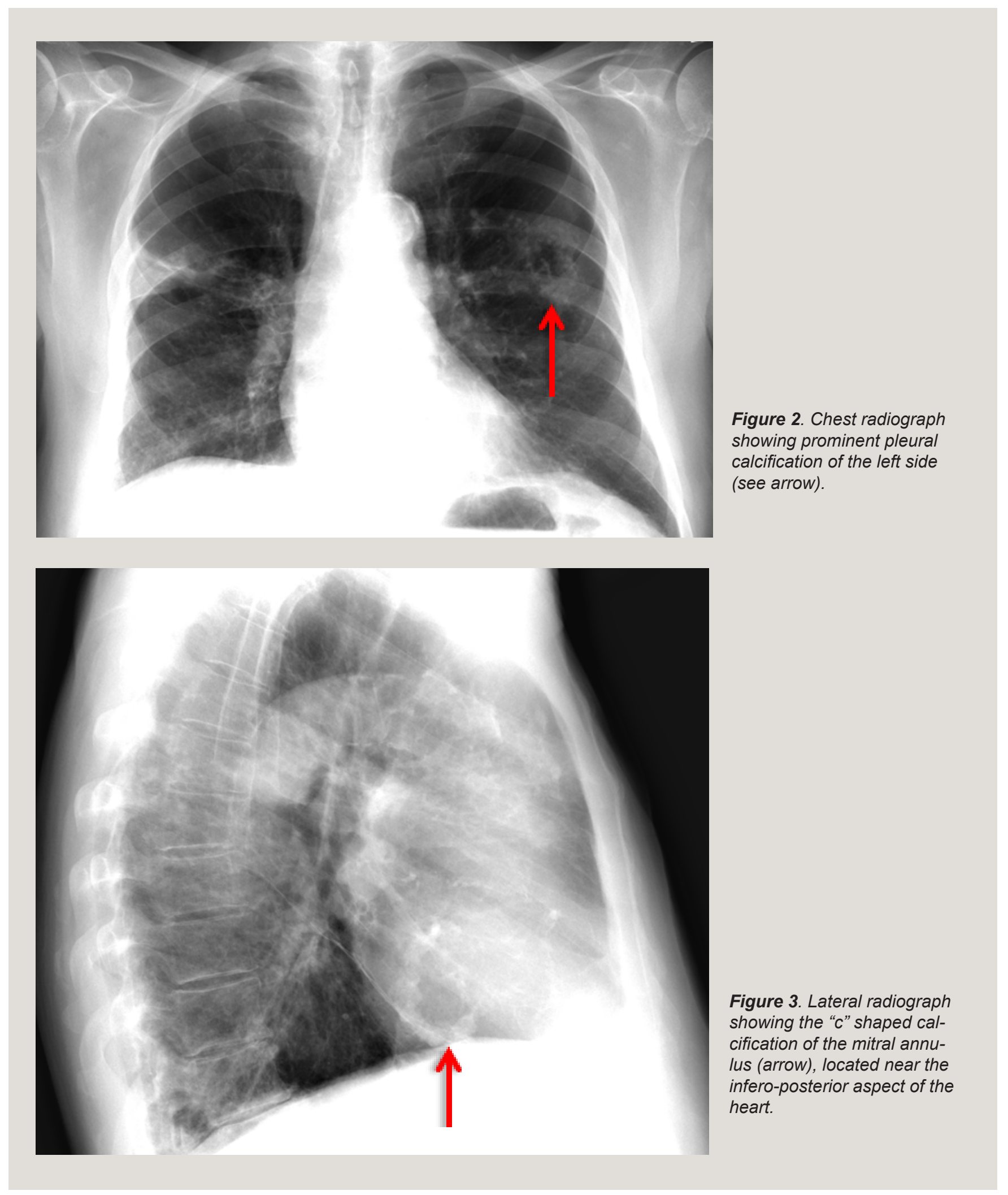


mid portion of the anterior mitral leaflet is in fibrous continuity with the aortic valve leaflets. Progressive deposition of calcium beneath and along the mitral annulus leads to mitral annular calcification. The calcification has a characteristic "c" shaped annulus sparing the base of the anterior mitral annulus and is more commonly found in patients older than 70 years and in women. ${ }^{1}$ Mitral annular calcification is usually diagnosed on echocardiograms and fluoroscopy but may be seen in radiographs as in our case. This is usually an incidental finding; it can rarely give rise to mitral regurgitation or mitral stenosis. Sparing of the anterior mitral leaflet and the valve commissures helps distinguish it from rheumatic mitral stenosis. Mitral annular calcification shares common risk factors with atherosclerosis and is associated with coronary artery disease, cardiac conduction system disease, and adverse cardiovascular events. ${ }^{2}$ It has been reported to have a positive predictive value of $92 \%$ for severe coronary artery disease in patients less than 65 years old. Our patient had diffuse vascular disease, including coronary disease.

Although pleural thickening of any etiology may later develop calcification, it is commonly due to infections, hemorrhage, or asbestos exposure. Calcifications due to hemorrhage and infections are usually unilateral and may vary in extent from minimal to massive. Our patient had a history of pneumonia in the past and possibly developed this after this infection. He did not have a history of asbestos exposure.

\section{References}

1. Fulkerson PK, Beaver BM, Auseon JC, et al. Calcification of the mitral annulus: aetiology, clinical associations, complications and therapy. Am J Med 1979; 66:967-77.

2. Adler Y, Zabarski RS, Vaturi M, et al. The association between mitral annulus calcium and aortic atheroma as detected by transesophageal echocardiographic study. Am J Cardiol $1998 ; 81: 784-6$.

Author affiliation : Ragesh Panikkath and Deepa Panikkath are internal medicine residents at TTUHSC in Lubbock.

Submitted: $2 / 7 / 2014$

Accepted: $2 / 25 / 2014$

Reviewers: Eman Attaya MD

Conflict of Interest: None

Published electronically: 4/15/2014 